\title{
FACTORES DETERMINANTES PARA EL ACCESO DE LAS MIPYME AL CRÉDITO GOTA A GOTA*
}

\section{Determining factors for MSME's access to credit drop by drop}

Jhony Alexander Barrera Lievano**

Sandra Miyey Parra Ramírez***

Recepción: 06/10/2019. Aceptación: 18/12/2019

DOI: http://dx.doi.org/10.21017/Rev.Repub.2020.v28.a84

\section{RESUMEN}

Esta investigación tiene como propósito identificar los factores determinantes que llevan a las micro, pequeñas y medianas empresas - Mipyme a tomar créditos gota a gota. Se desarrolla una investigación con metodología cuantitativa, de tipo exploratoria; se recolectan, procesan y analizan datos primarios; el instrumento de recolección de información a utilizar es la encuesta; se trabaja con una muestra probabilística, el tipo de muestreo es aleatorio simple. Se aplica el instrumento de recolección de datos a una muestra de 385 administradores de Mipyme de Bogotá DC - Colombia. Como principales hallazgos se identifica que los determinantes que llevan a la toma de éste tipo de créditos están asociados por un lado a características de las unidades empresariales en cuanto a tamaño de la empresa y nivel o grado de escolaridad del administrador, y por otro lado asociados a la percepción del administrador en cuanto a la aprobación del crédito (agilidad de desembolso, no tramitología y oferta a la mano).

* Artículo de investigación en colaboración que los autores desarrollar sobre el tema en el marco de un ejercicio de indagación y sistematización de tipo cuantitativo.

** Estudiante de Doctorado en Proyectos de la Universidad Americana de Europa (México); Magister en Dirección estratégica con especialidad en gerencia de la Universidad Internacional Iberoamericana (Estados Unidos); Especialista en Finanzas y Administrador de Empresas de la Corporación Universitaria Minuto de Dios (Colombia). Profesor de la Facultad de Finanzas y Comercio Internacional de la Corporación Universitaria Republicana. Correo electrónico: jbarrera@urepublicana.edu.co; jhonybarrera.lievano@ gmail.com

*** Magistra en Relaciones internacionales de la Pontificia Universidad Javeriana (Colombia); Especialista en Administración financiera de la Universidad EAN (Colombia); Profesional en Relaciones económicas internacionales de la Universidad Autónoma de Colombia (Colombia). Profesora Facultad de Ciencias Empresariales de la Corporación Universitaria Minuto de Dios - Sede UVD. Correo electrónico: sandra.parra@ uniminuto.edu 
Palabras clave: Crédito formal; crédito informal; crédito gota a gota; Mipyme JEL: G3; M2; K2

\begin{abstract}
The propose o this research is to identify the determining factors that lead micro, small and medium enterprises - MSME to take credits drop by drop. An investigation is carried out with quantitative methodology, of exploratory type; primary data is collected, processed and analyzed; the instrument for collecting information to use is the survey; it works with a probabilistic sample, the type of sampling is simple random. The data collection instrument is applied to a sample of 385 managers of Mipyme of Bogotá DC-Colombia. As main findings, it is identified that the determinants that lead to the taking of this type of credit are associated on the one hand with characteristics of the business units in terms of company size and level of education of the manager, and on the other hand associated to the perception of the manager regarding the approval of the credit (disbursement agility, no paperwork and offer at hand).
\end{abstract}

Key words: Formal credit; informal credit; drop by drop credit; MSME JEL: G3; M2; K2

\title{
1. INTRODUCCIÓN
}

El acceso al crédito en una economía juega un papel relevante y protagónico. $\mathrm{Su}$ tipificación y oferta suele estar regulada y reglamentada en cada país, sin importar el sistema o modelo económico que tenga implementado. Ya sea que el crédito sea ofrecido por el sector público o por el sector privado (y todas sus gamificaciones), este ejercicio procura la colocación de recursos monetarios con diversas finalidades.

De las líneas más estudiadas y tratadas de colocación de crédito, son las que van dirigidas al sector empresarial, y no es raro al comprender que las empresas, como parte del circuito económico dinamizan el funcionamiento de la economía, por las relaciones que genera con el Estado, con las familias y con otras empresas (Massad, 2007).

Las empresas en Colombia son clasificadas por diferentes variables para determinar su tamaño (Barrera Lievano, 2019). Según legislación vigente, estas unidades de negocio pueden ser clasificadas como microempresa, pequeña empresa, mediana empresa y empresa; las tres clasificaciones primeras, determinadas como Mipyme, son mayoría en el país, y generan la mayor cantidad de empleo (Melgarejo et al., 2013), así como también lo hacen a nivel mundial (Pinto, 2007). 
Aunque el crédito juega un papel importante en la economía, no todas las empresas, especialmente las de menor tamaño, pueden acceder a la oferta del mercado regular, por lo cual suplen sus necesidades de financiación con fuentes, que son de alto costo, de alto riesgo financiero, de alto riesgo social y de alto riesgo de seguridad, como por ejemplo los créditos gota a gota.

Con base en lo anterior surge la siguiente pregunta: ¿cuáles son los determinantes para que los micro, pequeños y medianos empresarios tomen crédito gota a gota? El identificar factores puede ayudar a la oferta formal crediticia a generar estrategias para aumentar el acceso al crédito a Mipyme. Se propone como objetivo de investigación identificar los factores determinantes que llevan a las Mipyme a tomar créditos gota a gota.

Para abordar esta pregunta se propone trabajar con la población de Mipyme que desarrollan sus actividades económicas en la Corporación de abastos de Bogotá S.A. - Corabastos, ubicada en la ciudad de Bogotá, en la localidad de Kennedy, según clasificación por tamaño de empresa a 31 de diciembre de 2018.

Se plantea el desarrollo de una investigación con metodología cuantitativa, de tipo exploratoria; se recolectan, procesan y analizan datos primarios; el instrumento de recolección de información a utilizar es la encuesta; se trabaja con una muestra probabilística, el tipo de muestreo es aleatorio simple.

\section{REVISIÓN LITERARIA}

El significado de empresa es amplio en la literatura existente a nivel de las ciencias sociales. Como lo asegura Barrera Lievano (2017: 29), después de realizar una revisión literaria frente a las definiciones de la palabra empresa en diferentes campos del conocimiento, ésta puede ser definida como un:

Sistema conformado por un grupo de personas que mediante la aplicación del proceso administrativo busca producir, distribuir y / o comercializar bienes y / o servicios, utilizando los factores de producción, en busca de un fin determinado dado por sus dueños o propietarios, que en todos los casos siempre será la obtención de riqueza económica. Este sistema se caracteriza por ser una unidad compleja y por estar inmersa en un ambiente de riesgo.

Las empresas en Colombia se clasifican, según tamaño, acorde a lo que determina la legislación. Sus clasificaciones son denominadas como microempresa, pequeña empresa, mediana empresa, y empresa. A 31 de diciembre de 2018 la legislación existente que reglamentó el tamaño de las empresas estuvo enmarcada en la ley 590 de 2000, la ley 905 de 2004, la ley 1151 de 2007, y la ley 1450 de 2011, las cuales establecen lo siguiente: 
Tabla 1. Tamaño de empresa (Mipyme) legislación vigente a 2018.

\begin{tabular}{|c|c|c|c|}
\hline $\begin{array}{c}\text { Tamaño de } \\
\text { empresa }\end{array}$ & $\begin{array}{c}\text { No. de } \\
\text { trabajadores }\end{array}$ & Total de activos & $\begin{array}{c}\text { Ventas brutas } \\
\text { anuales }\end{array}$ \\
\hline Micro & Menos de 11 & Menos de $501 \mathrm{smmlv}^{1}$ & No reglamentada \\
\hline Pequeña & Entre 11 y 50 & Entre 501 y $5.000 \mathrm{smmlv}$ & No reglamentada \\
\hline Mediana & Entre 51 y 200 & Entre 100.000 y $610.000 \mathrm{UVT}^{2}$ & No reglamentada \\
\hline
\end{tabular}

Fuente: adaptado de Ley 590 de 2000, Ley 905 de 2004, Ley 1151 de 2007, y Ley 1450 de 2011

Como se puede apreciar, son tres las variables que se toman en cuenta para la clasificación de las Mipyme según la legislación citada. Sin embargo, no todas las variables están reglamentadas a 2018. Como lo menciona Barrera (2019) la variable de "valor de ventas brutas anuales" planteada en la Ley 1151 de 2007 y ratificada en la Ley 1450 de 2011 a 2018 no había sido aún reglamentada.

Las empresas financian sus actividades a través de dos vías, bien sea con recursos provenientes del patrimonio de los socios, o con recursos de terceros, ya que como lo Fernandez (2014) el activo de las empresas, su reconocimiento, lleva a que en contrapartida haya un aumento del pasivo o del patrimonio. Las empresas determinan su estructura de financiación al incurrir en el uso de las fuentes ya mencionadas. Estas decisiones de financiamiento se pueden asociar a diferentes variables, entre otras el estilo de la dirección (Barton y Gordon, 1987, citados por Pesce, et al, 2015), el tamaño de la empresa (Botello, 2015), el impacto de los costos de agencia en la adquisición de deuda (Pesce, et al, 2015), e aprovechamiento de beneficios fiscales por tener deuda (Barrera et al., 2020), entre otros.

Ramírez (2009) menciona que la banca existe desde la época de los sumerios, entre el 3.400 y el 3.200 AC. La necesidad del mercado en cuanto a la existencia de instrumentos y mecanismos de ahorro, crédito e inversión, hacen necesaria la existencia de intermediarios financieros (Bhattacharya y Thakor, 1993) (Barrios, 2004) que cumplen el papel captación y colocación de recursos, y conforman el denominado sistema financiero.

Respecto al sistema financiero colombiano, el decreto 663 del 2 de abril de 1993 Por medio del cual se actualiza el Estatuto Orgánico del Sistema Financiero y se modifica su titulación y numeración establece en su artículo primero que la estructura general del sistema financiero colombiano se encuentra conformado por: establecimientos de crédito; sociedades de servicios financieros; sociedades de capitalización; entidades aseguradoras; e, intermediarios de seguros y

1 smmlv: salario mínimo mensual legal vigente

2 UVT: Unidad de Valor Tributario 
reaseguros. Según la Superintendencia financiera de Colombia (2019) la estructura del sistema financiero colombiano está conformada como se presenta en la tabla siguiente tabla.

Tabla 2. Sistema financiero colombiano.

\begin{tabular}{|c|c|c|}
\hline $\begin{array}{l}\text { Tipología de } \\
\text { la entidad/ } \\
\text { agente }\end{array}$ & Descripción general & Tipo de entidad \\
\hline \multirow{4}{*}{$\begin{array}{l}\text { Establecimien } \\
\text { tos de Crédito }\end{array}$} & \multirow{4}{*}{$\begin{array}{l}\text { Se consideran establecimientos de crédito } \\
\text { las instituciones financieras cuya función } \\
\text { principal consista en captar en moneda } \\
\text { legal recursos del público en depósitos a } \\
\text { la vista (cuentas de ahorro, corriente) o a } \\
\text { término (CDT y CDAT'S), para colocar- } \\
\text { los nuevamente a través de préstamos, } \\
\text { descuentos, anticipos u otras operaciones } \\
\text { activas de crédito. }\end{array}$} & Establecimientos Bancarios \\
\hline & & Corporaciones Financieras \\
\hline & & $\begin{array}{l}\text { Compañías de Financia- } \\
\text { miento Comercial }\end{array}$ \\
\hline & & Cooperativas Financieras \\
\hline \multirow{4}{*}{$\begin{array}{l}\text { Sociedades de } \\
\text { Servicios } \\
\text { Financieros }\end{array}$} & \multirow{4}{*}{$\begin{array}{l}\text { Son sociedades que tienen por función la } \\
\text { realización de las operaciones previstas } \\
\text { en el régimen legal que regula su acti- } \\
\text { vidad, si bien captan recursos del ahorro } \\
\text { público, por la naturaleza de su actividad } \\
\text { se consideran como instituciones que } \\
\text { prestan servicios complementarios y } \\
\text { conexos con la actividad financiera. }\end{array}$} & Sociedades Fiduciarias \\
\hline & & $\begin{array}{l}\text { Almacenes Generales de } \\
\text { Depósito }\end{array}$ \\
\hline & & $\begin{array}{l}\text { Sociedades } \\
\text { Administradoras de Pen- } \\
\text { siones y Cesantías }\end{array}$ \\
\hline & & $\begin{array}{l}\text { Sociedades de intermedia- } \\
\text { ción cambiaria y de servi- } \\
\text { cios financieros especiales, } \\
\text { abreviatura }\end{array}$ \\
\hline $\begin{array}{l}\text { Sociedades de } \\
\text { Capitalización }\end{array}$ & $\begin{array}{l}\text { Son instituciones financieras cuyo objeto } \\
\text { consiste en estimular el ahorro mediante } \\
\text { la constitución, en cualquier forma, de } \\
\text { capitales determinados, a cambio de } \\
\text { desembolsos únicos o periódicos, con } \\
\text { posibilidad o sin ella de reembolsos } \\
\text { anticipados por medio de sorteos. }\end{array}$ & \\
\hline \multirow{3}{*}{$\begin{array}{l}\text { Entidades } \\
\text { Aseguradoras }\end{array}$} & \multirow{3}{*}{$\begin{array}{l}\text { Su objeto es la realización de operaciones } \\
\text { de seguro, bajo las modalidades y los } \\
\text { ramos facultados expresamente. }\end{array}$} & Las Compañías de Seguros \\
\hline & & $\begin{array}{l}\text { Las Compañías de Re- } \\
\text { aseguros }\end{array}$ \\
\hline & & $\begin{array}{ll}\text { Las Cooperativas de } \\
\text { Seguros }\end{array}$ \\
\hline \multirow{3}{*}{$\begin{array}{l}\text { Intermediarios } \\
\text { de Seguros y } \\
\text { Reaseguros }\end{array}$} & & $\begin{array}{l}\text { Los corredores de Seguros } \\
\text { Las agencias de Seguros }\end{array}$ \\
\hline & & Los agentes de Seguros \\
\hline & & Corredores de Reaseguros \\
\hline
\end{tabular}

Fuente: adaptado de Superfinanciera (2019) 
La función principal de los establecimientos de crédito especificados es la de "canalizar recursos de los agentes superavitarios de la economía hacia los deficitarios, mediante la captación de fondos del público en moneda legal, para su posterior colocación por medio de préstamos y otras operaciones activas" (Escobar, 2013: 5). En términos el sistema financiero colombiano está clasificado como se presenta en la figura 1.
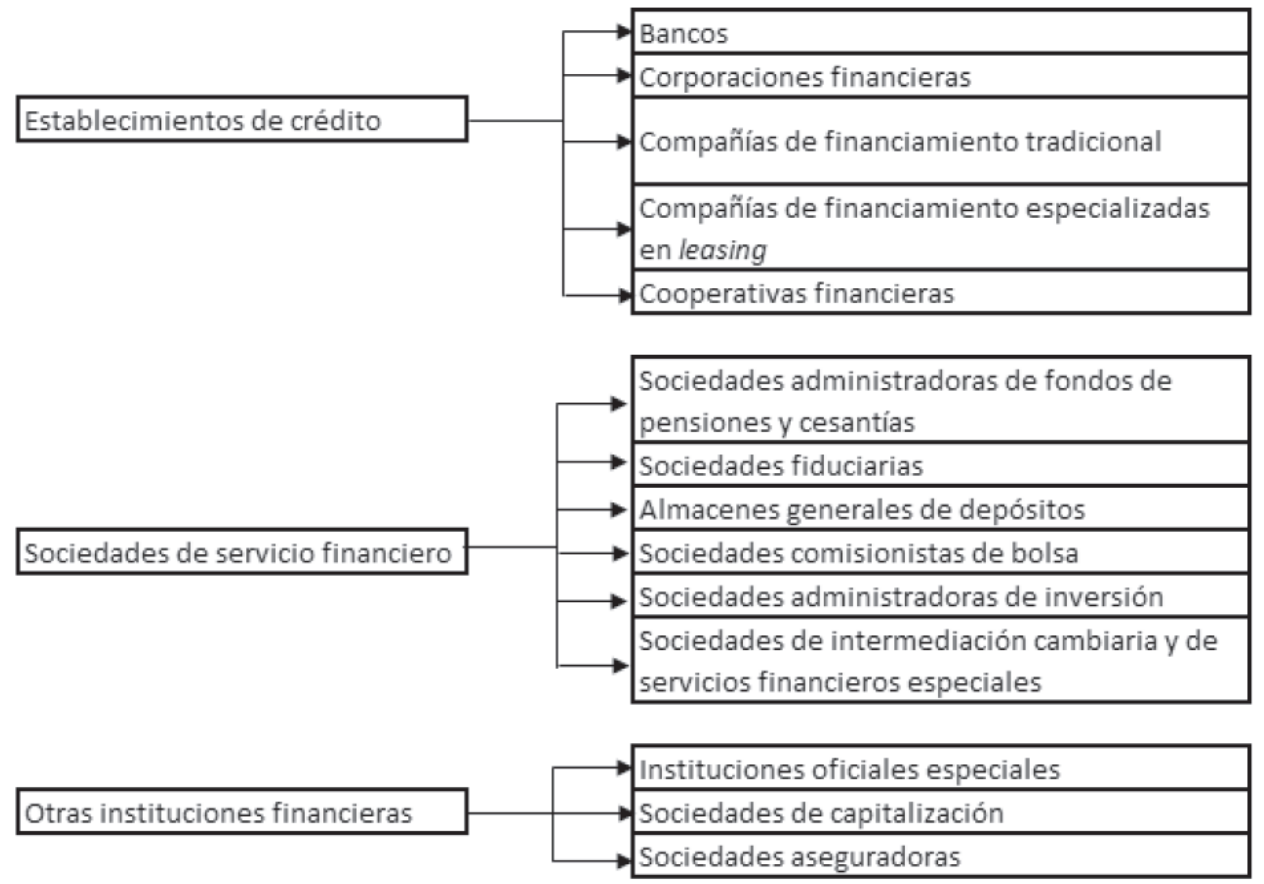

Fuente: Escobar, J. D. U. (2013, p. 6)

Figura 1. Clasificación entidades sistema financiero.

Un factor determinante del crecimiento de una economía es el acceso al sistema financiero que pueden tener las personas (naturales y jurídicas) que la conforman. Como lo mencionan Espinel y Giraldo (2009) la generación de ingreso y su distribución dentro de la población, son factores relevantes para el desempeño macroeconómico de un país. El acceso al crédito formal para las Mipyme en Colombia, sobre todo en las Pyme, está restringido en gran manera "debido a la dificultad que tienen para satisfacer los requisitos de documentación y garantías que el mercado impone, y porque las condiciones de los créditos no se ajustan a sus necesidades" (Montoya, 2001: 227). En Colombia, como en otros países de la región, existe una población: "cuyo acceso a los sistemas 
bancarios tradicionales es limitado o inexistente en virtud de su condición socioeconómica; es decir, a actores con bajos niveles de ingresos, consideraros como clientes no confiables por los bancos" (Mballa, 2017: 111).

Entre las condicionantes de acceso al crédito ofrecido por el sistema financiero formal que tienen las Mipyme se encuentran las garantías reales a comprometer (Cull et al., 2007); el costo del crédito y la capacidad de pago (Figueroa, 2016); la capacidad financiera, la pérdida no credibilidad en el sistema, las variaciones del mercado (Valencia, 2010: 130); la estabilidad económica del país y la cantidad de recursos que pueden captar las entidades financieras (Rosende, 1986).

Ante el vacío existente en el mercado formal en cuanto al acceso al crédito a las Mipyme, existen respuestas del mercado informal que atienden dichas necesidades (Ramirez-Urquid, et al. 2009). Al no lograr satisfacer las necesidades impuestas por las entidades del sistema financiero, las Mipyme acuden a fuentes informales de crédito (Montoya, 2001). El crédito informal es "es una actividad de intermediación realizada por prestamistas no especializados, que no cuenta con supervisión ni respaldo del Estado" (Trivelli, 1994, citado por García y Gómez 2016: 139).

El punto central del crédito informal está en la legalidad o ilegalidad que éste presente. El carácter de ilegalidad del crédito informal está ligado no a la informalidad, aunque la informalidad en ocasiones puede ser vista como un delito. Como lo mencionan Mauricio y Sandra (2009: 273) en Colombia se puede entender por informalidad "no contar con un registro mercantil". Una persona que ofrezca un crédito informal en primera instancia no está incurriendo en un delito por el hecho de no contar con registro mercantil. Ejemplo de ello puede ser el préstamo de un familiar.

El carácter de ilegalidad del crédito informal lo puede dar los métodos de coacción que se ejerzan en el cobro del mismo, o la tasa de interés que se cobre sobre éste. En cuanto al recaudo, el retraso en el pago de las cuotas pactadas en el sistema informal puede llevar a "desplazamiento del lugar donde residen; pérdida del negocio; los familiares heredan las deudas, poniendo a su vez en riesgo la vida de ellos; homicidio del deudor o familiares cercanos" (García y Gómez, 2016: 138). Respecto a la tasa de interés, para el caso específico de Colombia, la ley 599 del año 2000 especifica en su artículo 305 (modificado por la ley 890 de 2004) que:

El que reciba o cobre, directa o indirectamente, a cambio de préstamo de dinero o por concepto de venta de bienes o servicios a plazo, utilidad o ventaja que exceda en la mitad del interés bancario corriente que para el 
período correspondiente estén cobrando los bancos, según certificación de la Superintendencia Bancaria, cualquiera sea la forma utilizada para hacer constar la operación, ocultarla o disimularla, incurrirá en prisión de treinta y dos (32) a noventa (90) meses y multa de sesenta y seis punto sesenta y seis (66.66) a trescientos (300) salarios mínimos legales mensuales vigentes.

El crédito gota a gota, también denominado crédito cuentagotas, puede ser definido como un crédito a ser pagado a través de pequeñas cantidades de dinero, con una frecuencia de pago relativamente más corta que la que ofrece la banca tradicional (García, et al, 2017). Esta frecuencia de pago puede ser diaria, semanal, entre otras que puedan definir los pactantes (Holguín, 2018). Las altas tasas de interés asociadas a este tipo de crédito pueden oscilar entre la superación de la tasa mínima estipulada por la ley 599 del año 2000 artículo 305, y hasta tasas mensuales del 15\% (Asobancaria, 2019). La tasa del 15\% mensual en términos efectivos anuales es equivalente a $435,03 \%$. Ahora, hay quienes aseguran que la tasa máxima puede ser más elevada, caso de Holguín (2018) quien afirma que esta puede ser hasta del $20 \%$ diario.

\section{CONTEXTUALIZACIÓN}

Según la información oficial publicada en su página Web (Corabastos, 2019a), la Corporación de abastos de Bogotá S.A. - Corabastos tiene sus orígenes en el año de 1970, y fue creada con el objetivo de: "organizar el sistema de mercadeo de alimentos y centralizar y racionalizar su distribución. Como entidad es una Sociedad del orden nacional, de economía mixta vinculada al Ministerio de Agricultura y Desarrollo Rural".

De igual forma, la misma fuente especifica que, en total, Corabastos cuenta con 420.000 metros cuadrados, en donde funcionan 57 bodegas, y 16 entidades bancarias. En total cuenta con 6.500 empresarios mayoristas y minoristas; en sus instalaciones de manera diaria circulan en promedio 200.000 personas, y abastece en términos de cobertura a 10 millones de personas. Corabastos moviliza diariamente más de 12.400 toneladas de alimentos y maneja unas ventas diarias del orden aproximado de COP \$24.000 millones (Corabastos, 2019a).

Aunque en la información de "Nuestra Historia", registrada en la página Web de Corabastos, se especifica que cuenta con 57 bodegas; al ingresar al directorio comercial, y al verificar los locales registrados, se identifica que Corabastos cuenta con 62 bodegas, que albergan un total de 5.152 locales (Corabastos, $2019 b$ ), con un rango de total de negocio por bodega que oscila entre 1 y 1.420 . Esta diferencia lleva a que no se cuente con un dato exacto del total del parque empresarial instalado allí. 


\section{METODOLOGÍA DEL ESTUDIO}

Para dar respuesta a la pregunta de investigación, se desarrolla una investigación con metodología cuantitativa. El tipo de investigación es exploratoria (Hernandez et al., 2010). El instrumento de recolección de información a utilizar es la encuesta. La encuesta diseñada presenta solo preguntas cerradas. La alimentación de la encuesta es totalmente anónima. La validación de este instrumento se realiza con dueños o administradores de Mipyme de la zona, para determinar que el vocabulario empleado sea el adecuado para la población objetivo, debido a la disparidad en la formación académica que se puede presentar entre los diferentes administradores de Mipyme de Corabastos. Respecto a la aplicación del instrumento de recolección de información de datos primarios, se aplica a una muestra determinada según la cantidad de negocios existentes dentro de Corabastos, con las siguientes variables: Tamaño de la población, desconocido ${ }^{3}$; heterogeneidad, $50 \%$; nivel de confianza, $95 \%$; margen de error del $5 \%$.

Para calcular la muestra se parte de la base del no conocimiento del total la población. Al tener la estimación del nivel de confianza y determinar el margen de error, se aplica la siguiente fórmula (Badii et al., 2017):

$$
n=\frac{z^{2} p q}{d^{2}}
$$

Donde:

$\mathrm{z}$ = Factor de distribución normal con base al nivel de confianza

$\mathrm{p}=$ Probabilidad de que ocurra el evento

$\mathrm{q}$ = Probabilidad de que no ocurra el evento

$\mathrm{d}=$ Margen de error

De tal forma que:

$$
n=\frac{1,96^{2} * 0,5 * 0,5}{0,05^{2}} \quad n=\frac{3,8416 * 0,5 * 0,5}{0,0025} \quad n=\frac{0,9604}{0,0025}=384,16=385
$$

Tipo de muestreo, probabilístico, aleatorio simple, proporcional a la cantidad de negocios que se encuentran registrados por bodega. Esto significa que, la cantidad de encuestas que se aplica en cada bodega es proporcional a la cantidad

3 Aunque se conoce el total del empresarios mayoristas y minoristas, y el total de locales en todas las bodegas, no se conoce de ellos cuantos son dueños o administradores de Mipyme, por lo cual no se conoce el total de la población a la cual va dirigida la investigación. 
de puestos que se encuentra en cada una, con respecto al total de las encuestas a aplicar. La encuesta solo se le aplicará a Mipyme. Para que todos tengan probabilidad de ser seleccionados las encuestas se aplicarán cada 3 locales (donde aplique por cantidad), iniciando desde el número 1, moviéndose un local cada vez que se llegue a un negocio que manifieste no ser Mipyme, o que no atienda al desarrollo de la encuesta. En caso de que se dé la vuelta completa a la bodega, se iniciará nuevamente el recorrido iniciando en el local número dos, y así sucesivamente.

Para organización y análisis de la información se utilizará el sistema Statistical Package for the Social Sciences - SPSS versión 23.

\section{RESULTADOS}

Acorde a lo estipulado en la metodología, se aplicaron 385 encuestas a dueños o administradores de Mipyme de Corabastos. El 100\% de los encuestados fueron personas mayores de edad. Los resultados fueron los siguientes.

Del 100\% de los encuestados, el 79,5\% fueron hombres, el 20,5\% fueron mujeres y ningún encuestado especificó un género diferente. Respecto al nivel de formación, se encontró que entre el total de los encuestados se presentó una diversidad alta, como se presenta a continuación:

Tabla 3. Máximo nivel de formación

\begin{tabular}{|l|l|c|c|c|c|}
\hline \multicolumn{2}{|c|}{} & Frequency & Percent & Valid Percent & $\begin{array}{c}\text { Cumulative } \\
\text { Percent }\end{array}$ \\
\hline \multirow{4}{*}{ Valid } & Primaria & 57 & 14,8 & 14,8 & 14,8 \\
\cline { 2 - 6 } & Bachillerato & 165 & 42,9 & 42,9 & 57,7 \\
\cline { 2 - 6 } & Técnico & 37 & 9,6 & 9,6 & 67,3 \\
\cline { 2 - 6 } & Tecnólogo & 58 & 15,0 & 15,0 & 82,3 \\
\cline { 2 - 6 } & Profesional & 53 & 13,8 & 13,8 & 96,1 \\
\cline { 2 - 6 } & Postgrado & 10 & 2,6 & 2,6 & 98,7 \\
\cline { 2 - 6 } & Ninguno & 5 & 1,3 & 1,3 & 100,0 \\
\cline { 2 - 6 } & Total & 385 & 100,0 & 100,0 & \\
\hline
\end{tabular}

Fuente: creación propia.

Como se puede evidenciar, el 42,9\% registra como máxima formación el bachi1 lerato ${ }^{4}$, seguido del grado de tecnólogo $(15 \%)$, primaria $(14,8 \%)$, y profesional $(13,8 \%)$. Sin ningún tipo de formación, o analfabetismo, se evidencia un 1,3\%.

4 Educación segundaria o preparatoria. 
Con base al género del encuestado, y su nivel de formación, como se puede evidenciar en la figura 3 , tanto hombres como mujeres presentan respuestas en todas las opciones de nivel de formación, siendo desde ningún nivel de educación formal, hasta posgrado.

Tabla 4. Género y máximo nivel de formación.

\begin{tabular}{|c|c|c|c|c|c|c|c|c|c|}
\hline & \multicolumn{8}{|c|}{ Seleccione su máximo nivel de formación (marque solo una) } \\
\hline & & Primaria & Bachillerato & Técnico & Tecnólogo & Profesional & Posgrado & Ninguno & Total \\
\hline \multirow{2}{*}{ 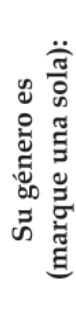 } & 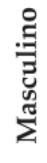 & 47 & 142 & 27 & 39 & 41 & 6 & 4 & 306 \\
\hline & 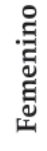 & 10 & 23 & 10 & 19 & 12 & 4 & 1 & 79 \\
\hline \multicolumn{2}{|c|}{ Total } & 57 & 165 & 37 & 58 & 53 & 10 & 5 & 385 \\
\hline
\end{tabular}

Fuente: creación propia.

Respecto al tiempo de funcionamiento de la Mipyme, el 54,5\% presenta una antigüedad mayor a o igual a 5 años, mientas que solo el 2,1\% presentó una antigüedad de menos de un año, como se presenta a continuación:

Tabla 5. Tiempo de funcionamiento de la Mipyme

\begin{tabular}{|l|l|c|c|c|c|}
\hline \multicolumn{2}{|l|}{} & Frequency & Percent & Valid Percent & $\begin{array}{c}\text { Cumulative } \\
\text { Percent }\end{array}$ \\
\hline \multirow{7}{*}{ Valid } & $\begin{array}{l}\text { Menos de un } \\
\text { año }\end{array}$ & 8 & 2,1 & 2,1 & 2,1 \\
\cline { 2 - 6 } & $\begin{array}{l}\text { Entre 1 y menos } \\
\text { de 3 años }\end{array}$ & 60 & 15,6 & 15,6 & 17,7 \\
\cline { 2 - 6 } & $\begin{array}{l}\text { Entre 3 y menos } \\
\text { de 5 años }\end{array}$ & 107 & 27,8 & 27,8 & 45,5 \\
\cline { 2 - 6 } & Entre 5 y 10 años & 128 & 33,2 & 33,2 & 78,7 \\
\cline { 2 - 6 } & Más de 10 años & 82 & 21,3 & 21,3 & 100,0 \\
\cline { 2 - 6 } & Total & 385 & 100,0 & 100,0 & \\
\hline
\end{tabular}

Fuente: creación propia.

En cuanto al tamaño de la Mipyme, según clasificación por ley, la mayor concentración se encontró en microempresas, seguidas de las pequeñas empresas y finalmente las medianas empresas, como lo presenta la tabla 6. 
Tabla 6. Clasificación por tamaño (a 2018)

\begin{tabular}{|l|l|c|c|c|c|}
\hline \multicolumn{2}{|c|}{} & Frequency & Percent & Valid Percent & $\begin{array}{c}\text { Cumulative } \\
\text { Percent }\end{array}$ \\
\hline \multirow{3}{*}{ Valid } & Microempresa & 273 & 70,9 & 70,9 & 70,9 \\
\cline { 2 - 6 } & Pequeña empresa & 72 & 18,7 & 18,7 & 89,6 \\
\cline { 2 - 6 } & Mediana empresa & 40 & 10,4 & 10,4 & 100,0 \\
\cline { 2 - 6 } & Total & 385 & 100,0 & 100,0 & \\
\hline
\end{tabular}

Del total de los encuestados, el 90,2\% de las empresas presentan registro mercantil activo, mientras el 9,8\% no presenta esta documentación. Respecto a si para la Mipyme se ha(n) tomado crédito(s) gota a gota, el 21,6\% manifestó haber tomado este tipo de créditos, mientras que un $78,4 \%$ dieron respuesta negativa a esta pregunta, como se presenta en la tabla 7.

Tabla 7. Afirmación o negación toma de crédito gota a gota para la Mipyme.

\begin{tabular}{|l|l|c|c|c|c|}
\hline \multicolumn{2}{|c|}{} & Frequency & Percent & Valid Percent & $\begin{array}{c}\text { Cumulative } \\
\text { Percent }\end{array}$ \\
\hline \multirow{3}{*}{ Valid } & Sí & 83 & 21,6 & 21,6 & 21,6 \\
\cline { 2 - 6 } & No & 302 & 78,4 & 78,4 & 100,0 \\
\cline { 2 - 6 } & Total & 385 & 100,0 & 100,0 & \\
\hline
\end{tabular}

Fuente: creación propia.

En cuanto a la relación de las Mipyme que han tomado crédito(s) gota a gota, y el género del dueño o administrador de la unidad empresarial, los resultados fueron los siguientes:

Tabla 8. Relación de género del dueño o administrador de la Mipyme y toma de crédito gota a gota

\begin{tabular}{|l|l|c|c|c|}
\hline \multicolumn{2}{|c|}{} & \multicolumn{2}{|c|}{$\begin{array}{c}\text { Ha tomado para su negocio crédito(s) gota a } \\
\text { gota (marque solo una) }\end{array}$} & Total \\
\cline { 3 - 5 } \multicolumn{2}{|c|}{} & Sí & No & \\
\hline \multirow{2}{*}{$\begin{array}{l}\text { Su género es } \\
\text { (marque una sola): }\end{array}$} & Masculino & 64 & 642 & 306 \\
\cline { 2 - 5 } & Femenino & 19 & 302 & 79 \\
\hline \multicolumn{2}{|l|}{ Total } & 83 & 605 \\
\hline
\end{tabular}

Fuente: creación propia.

Como se puede evidenciar, en proporción, el $24 \%$ del total de las mujeres encuestadas han accedido a créditos de gota a gota para la Mipyme de la cual son administradoras. Respecto a los hombres, el 20,9\% de los encuestados manifiestan haber realizado este mismo proceso.

Con base al nivel de formación del dueño o administrador de la Mipyme y la toma de crédito(s) gota a gota, según las respuestas obtenidas para las encuestas aplicadas, se encontró: 
Tabla 9. Relación máximo nivel de formación del dueño o administrador de la Mipyme y toma de crédito gota a gota.

\begin{tabular}{|c|c|c|c|c|}
\hline & \multicolumn{2}{|c|}{$\begin{array}{c}\text { Ha tomado para su negocio crédito(s) gota } \\
\text { a gota (marque solo una) }\end{array}$} & \multirow[t]{2}{*}{ Total } \\
\hline & & Sí & No & \\
\hline \multirow{7}{*}{$\begin{array}{l}\text { Seleccione su } \\
\text { máximo nivel de } \\
\text { formación } \\
\text { (marque solo } \\
\text { una) }\end{array}$} & Primaria & 23 & 34 & 57 \\
\hline & Bachillerato & 35 & 130 & 165 \\
\hline & Técnico & 8 & 29 & 37 \\
\hline & Tecnólogo & 6 & 52 & 58 \\
\hline & Profesional & 6 & 47 & 53 \\
\hline & Postgrado & 2 & 8 & 10 \\
\hline & Ninguno & 3 & 2 & 5 \\
\hline \multicolumn{2}{|l|}{ Total } & 83 & 302 & 385 \\
\hline
\end{tabular}

Fuente: Creación propia

Como se puede apreciar, de la población que responde que no tiene estudios, el $60 \%$ ha accedido a créditos de gota a gota para la Mipyme, mientras que quienes tiene como máximo nivel de formación el grado de primaria, el 40,35\% ha accedido a créditos gota a gota para la Mipyme. Respecto a los niveles de educación formal superiores al grado de primaria, en todos los niveles se encontró que (siendo administradores de la empresa) han accedido a créditos gota a gota; sin embargo, del total de la población para cada nivel, la toma de este tipo de créditos está entre el 10,34\% y el 21,62\%.

Respecto a la toma de crédito(s) gota a gota y tiempo de funcionamiento de la Mipyme, como se presenta en la tabla 10, se puede evidenciar que, superado el primer año de funcionamiento, entre el periodo de uno a menos de tres años

Tabla 10. Relación tiempo de funcionamiento de la Mipyme y toma de crédito gota a gota.

\begin{tabular}{|c|c|c|c|c|}
\hline & \multicolumn{2}{|c|}{$\begin{array}{c}\text { Ha tomado para su } \\
\text { negocio crédito(s) gota a } \\
\text { gota (marque solo una) }\end{array}$} & \multirow[t]{2}{*}{ Total } \\
\hline & & Sí & No & \\
\hline \multirow{5}{*}{$\begin{array}{l}\text { El tiempo de } \\
\text { funcionamiento } \\
\text { de su negocio } \\
\text { es de (marque } \\
\text { solo una) }\end{array}$} & Menos de un año & 1 & 7 & 8 \\
\hline & Entre 1 y menos de 3 años & 21 & 39 & 60 \\
\hline & Entre 3 y menos de 5 años & 19 & 88 & 107 \\
\hline & Entre 5 y 10 años & 22 & 106 & 128 \\
\hline & Más de 10 años & 20 & 62 & 82 \\
\hline \multicolumn{2}{|l|}{ Total } & 83 & 302 & 385 \\
\hline
\end{tabular}

Fuente: creación propia. 
de funcionamiento, las Mipyme encuestadas demandan mayor cantidad de créditos gota a gota, siendo un $35 \%$ del total de esta población quienes acceden a este tipo de créditos. De igual forma se evidencia que un alto porcentaje de Mipyme de más de 10 años de funcionamiento (24,39\%) acceden también a este tipo de créditos.

Respecto al tamaño de la Mipyme y la toma de crédito(s) gota a gota, se identifica que a menor tamaño hay mayor cantidad de tomadores de este tipo de créditos. Como se puede apreciar en la tabla 11, del 100\% de microempresas el $24,54 \%$ ha tomado crédito(s) gota a gota; del $100 \%$ de pequeñas empresas el $20,83 \%$ ha tomado crédito(s) gota a gota, y del 100\% de medianas empresas el $2,5 \%$ ha tomado crédito(s) gota a gota.

Tabla 11. Relación tamaño de la Mipyme y toma de crédito gota a gota.

\begin{tabular}{|c|c|c|c|c|}
\hline & \multicolumn{2}{|c|}{$\begin{array}{l}\text { Ha tomado para su } \\
\text { negocio crédito(s) gota a } \\
\text { gota (marque solo una) }\end{array}$} & \multirow[t]{2}{*}{ Total } \\
\hline & & Sí & No & \\
\hline \multirow{3}{*}{$\begin{array}{l}\text { A } 2018 \text { su } \\
\text { negocio estuvo } \\
\text { clasificado } \\
\text { como (marque } \\
\text { solo una) } \\
\end{array}$} & Microempresa & 67 & 206 & 273 \\
\hline & Pequeña empresa & 15 & 57 & 72 \\
\hline & Mediana empresa & 1 & 39 & 40 \\
\hline \multicolumn{2}{|l|}{ Total } & 83 & 302 & 385 \\
\hline
\end{tabular}

Fuente: creación propia.

Se le preguntó al 100\% de los encuestados respecto a qué asocian el crédito gota a gota, dándoles la posibilidad de seleccionar varias opciones entre una parrilla de 25 opciones totales. Los resultados se registran en la tabla 12.

Como se puede evidenciar, el mayor porcentaje de encuestados asegura asociar el crédito gota a gota con la palabra ilegal, $51,9 \%$, seguido de engaño con un $51,2 \%$, robo con un $49,6 \%$, interés alto con un $23,4 \%$. Por otra parte, algunos encuestados asocian el crédito gota a gota con palabras como ahorro (1\%), legal $(1 \%)$, o favor $(9,1 \%)$.

Finalmente, respecto a las variables que han llevado a que las personas encuestadas haya(n) tomado crédito(s) gota a gota para la Mipyme de la cual son dueños o administradores, al preguntarles a los encuestados por las causas que los han llevado a tomar este tipo de créditos, las respuestas dadas fueron las siguientes: 
Tabla 12. Palabras con que se asocia el crédito gota a gota.

\begin{tabular}{|c|c|c|c|}
\hline \multirow{2}{*}{\multicolumn{2}{|c|}{\begin{tabular}{|l|l|}
\multicolumn{2}{|c|}{} \\
$A$ cociación con & A gilidad
\end{tabular}}} & \multirow{2}{*}{$\frac{\text { Responses }}{37}$} & \multirow{2}{*}{$\begin{array}{c}\text { Percent over } \mathbf{N}^{*} \\
9,6\end{array}$} \\
\hline \multirow{24}{*}{$\begin{array}{l}\text { Asociación con } \\
\text { significado de } \\
\text { crédito gota a } \\
\text { gota }\end{array}$} & & & \\
\hline & Ahorro & 4 & 1,0 \\
\hline & Ayuda & 28 & 7,3 \\
\hline & Banco & 13 & 3,4 \\
\hline & Codeudor / Fiador & 18 & 4,7 \\
\hline & Cooperativa & 4 & 1,0 \\
\hline & Difícil acceso & 2 & 0,5 \\
\hline & Dinero & 47 & 12,2 \\
\hline & Discriminación & 5 & 1,3 \\
\hline & Engaño & 197 & 51,2 \\
\hline & Fácil acceso & 77 & 20,0 \\
\hline & Facilidad & 72 & 18,7 \\
\hline & Favor & 35 & 9,1 \\
\hline & Financiación & 21 & 5,5 \\
\hline & Hipoteca & 37 & 9,6 \\
\hline & Ilegal & 200 & 51,9 \\
\hline & Impuestos & 16 & 4,2 \\
\hline & Interés alto & 90 & 23,4 \\
\hline & Interés bajo & 4 & 1,0 \\
\hline & Legal & 4 & 1,0 \\
\hline & Pagaré & 17 & 4,4 \\
\hline & Papeleo & 21 & 5,5 \\
\hline & Robo & 191 & 49,6 \\
\hline & Tramitología & 6 & 1,6 \\
\hline
\end{tabular}

Fuente: creación propia.

Se evidencia que la agilidad en la entrega del dinero se convierte en la causa que mayor relevancia tiene a la hora de acceder a un crédito gota a gota $(39,8 \%)$, seguida de la aprobación inmediata $(36,1 \%)$, y de que no requiere trámites $(32,5 \%)$.

\section{DISCUSIÓN Y CONCLUSIONES}

El crédito es una de las principales fuentes de financiación de la empresa. De hecho, la estructura de capital de cualquier empresa proviene bien sea del patrimonio aportado por los dueños socios o accionistas (y de los resultados de los ejercicios de periodos anteriores), y del pasivo, que es el reconocimiento de deudas con terceros. El desempeño empresarial, el rendimiento económico-financiero, es una variable relevante para los dueños, socios o accionistas de las empresas. 
Tabla 13. Causas por las cuales el dueño o administrador de la Mipyme toma crédito(s) gota a gota.

\begin{tabular}{|c|c|c|c|}
\hline \multirow{5}{*}{\begin{tabular}{|l|} 
Causas por \\
las cuales \\
ha tomado \\
préstamos \\
gota a gota
\end{tabular}} & \multirow{2}{*}{ Agilidad en la entrega del dinero } & \multirow{2}{*}{$\frac{\text { Responses }}{33}$} & \multirow{2}{*}{$\frac{\text { Percent over } \mathbf{N}^{*}}{39,8}$} \\
\hline & & & \\
\hline & Aprobación inmediata & 30 & 36,1 \\
\hline & $\begin{array}{l}\text { Desembolso de montos pequeños, } \\
\text { medianos y grandes }\end{array}$ & 4 & 4,8 \\
\hline & $\begin{array}{l}\text { Es más seguro que un banco o una } \\
\text { entidad financiera }\end{array}$ & 2 & 2,4 \\
\hline & $\begin{array}{l}\text { La tasa de interés es más baja que en } \\
\text { el sistema bancario }\end{array}$ & 3 & 3,6 \\
\hline & $\begin{array}{l}\text { Los bancos o entidades financieras no } \\
\text { le prestan dinero }\end{array}$ & 12 & 14,5 \\
\hline & No requiere fiador & 16 & 19,3 \\
\hline & No requiere trámites & 27 & 32,5 \\
\hline & $\begin{array}{l}\text { Pago de cuotas en su negocio o } \\
\text { residencia }\end{array}$ & 7 & 8,4 \\
\hline & $\begin{array}{l}\text { Porque la oferta está a la mano y no } \\
\text { debe ir a ninguna otra parte }\end{array}$ & 18 & 21,7 \\
\hline & $\begin{array}{l}\text { Posibilidad de selección de frecuencia } \\
\text { de pago }\end{array}$ & 13 & 15,7 \\
\hline & $\begin{array}{l}\text { Puede acceder varias veces al crédito } \\
\text { en el año }\end{array}$ & 11 & 13,3 \\
\hline & $\begin{array}{l}\text { Tiempo de un mes o menos para } \\
\text { pagarlo }\end{array}$ & 12 & 14,5 \\
\hline & Tramitología & 5 & 6,0 \\
\hline
\end{tabular}

Fuente: creación propia.

Aplicado el instrumento de recolección de información, dirigido a Mipyme (según clasificación vigente a 2018) que desarrollan sus actividades económicas en Corporación de abastos de Bogotá S.A. - Corabastos, a una muestra que tiende al infinito con un nivel de confianza del 95\% y un margen de error del $5 \%$, se encontró que el 21,6\% de los administradores de las Mipyme aseguran que han accedido a créditos de gota a gota dentro del desarrollo de sus actividades empresariales.

Del 100\% de los encuestados, el 79,48\% fueron hombres y el restante 20,52\% mujeres. Esto deja una relación de hombre / mujer de todos los encuestados de 3,87 hombres por cada mujer encuestada. El 20,84\% de los hombres administradores de Mipyme encuestados aseguran haber tomado crédito(s) gota a gota para su unidad empresarial, mientras que, para el género femenino, este hecho representó el 24,05\%, siendo mayor que el de los hombres en 3,21 puntos porcentuales. 
Respecto al nivel de escolaridad, se encontró que, en términos relativos, a menor nivel de formación mayor cantidad de población accede a este tipo de créditos; es así como se evidenció que de la población que respondió no tener estudios, el $60 \%$ ha accedido a créditos de gota a gota para la Mipyme de la cual son administradores; la población que respondió tener como máximo nivel de formación el grado de primaria, el $40,35 \%$ ha accedido a este tipo de créditos en las mismas condiciones planteadas. Respecto a los niveles de educación superiores al grado de primaria, en todos los niveles se encontró que han accedido a créditos gota a gota, sin embargo, del total de la población para cada nivel, la toma de este tipo de créditos está entre el 10,34\% y el $21,21 \%$.

Respecto al tamaño de la empresa según clasificación vigente a 2018, se identificó que mientras menor sea el tamaño de la Mipyme, existe mayor propensión a tomar créditos gota a gota. Del 100\% de microempresas el $24,45 \%$ ha tomado crédito(s) gota a gota, del 100\% de pequeñas empresas el 20,83\% ha tomado crédito(s) gota a gota, y del 100\% de medianas empresas el 2,5\% ha tomado crédito(s) gota a gota.

Respecto a los determinantes que llevan a que micro, pequeños y medianos empresarios tomen crédito gota a gota, particularmente en Corabastos, se encontró lo que se resumen en la tabla 14:

Tabla 14. Determinantes para que los micro, pequeños y medianos empresarios tomen crédito gota a gota.

\begin{tabular}{|c|c|}
\hline $\begin{array}{c}\text { Asociados a las características de la } \\
\text { Mipyme }\end{array}$ & $\begin{array}{c}\text { Asociados a la percepción del administrador } \\
\text { de la Mipyme }\end{array}$ \\
\hline $\begin{array}{l}\text { Tamaño de la empresa (A menor } \\
\text { tamaño mayor propensión a tomar } \\
\text { créditos gota a gota) } \\
\text { Nivel o grado de escolaridad del } \\
\text { administrador (A menor nivel o grado } \\
\text { de escolaridad mayor propensión a } \\
\text { tomar créditos gota a gota) }\end{array}$ & $\begin{array}{l}\text { Aprobación inmediata del crédito gota a gota } \\
\text { Agilidad en la entrega del dinero del crédito } \\
\text { gota a gota } \\
\text { Que no se requieran trámites } \\
\text { Que la oferta esté a la mano y no deba ir a otra } \\
\text { parte }\end{array}$ \\
\hline
\end{tabular}

Fuente: creación propia.

Se proponen como futuros temas de investigación determinar la asociación que exista entre las características de las Mipyme y el acceso al crédito gota a gota, así como la correlación que pueda existir entre estas para determinar dirección y fuera o grado de significancia. 


\section{REFERENCIAS}

Asobancaria (2019). “Los peligros de los préstamos gota a gota”, Disponible en: https:/ /www.sabermassermas.com/los-peligros-de-los-prestamos-gota-a-gota/ recuperado: 22 de enero de 2020

Badii, M. H., Castillo, J., \& Guillen, A. (2017). "Tamaño óptimo de la muestra", En: InnOvaciOnes de NegOciOs, vol. 5 núm. 9. [En línea] Disponible en: http:// revistainnovaciones.uanl.mx/index.php/revin/article/viewFile/199/184 recuperado: 22 de enero de 2020

Barrera Lievano, J. (2017). Organizaciones y empresas. Relación, diferencias y clasificaciones, Saarbrücken, Editorial Académica Española

Barrera Lievano J. (2019). “Juntas de acción comunal y pequeñas y medianas empresas ¿articulación para el desarrollo de actividades de responsabilidad social empresarial", En: Tendencias, vol. 20, núm. 1. [En línea] Disponible en: https:// doi.org/10.22267/rtend.192001.107 recuperado: 22 de enero de 2020

Barrera Lievano, J., Parada, S., \& Serrano, L. (2020).” Análisis empírico de correlación entre el indicador de estructura de capital y el indicador de margen de utilidad neta en pequeñas y medianas empresas - PYME". En: Revista de Métodos Cuantitativos para la Economía y la Empresa, vol. 29, núm. 2.

Barrios Pérez, V. E. (2004) “¿Por qué existen los bancos?”, En: Boletín económico de ICE, núm. 2799. [En línea] Disponible en https://dialnet.unirioja.es/ejemplar/ 89395 recuperado: 22 de enero de 2020

Bhattacharya, S., \& Thakor, A. V. (1993). "Contemporary banking theory", En: Journal of financial Intermediation, vol. 3, núm. 1, [En línea] Disponible en https:/ / doi.org/ 10.1006/jfin.1993.1001 recuperado: 22 de enero de 2020

Botello, H. (2015). "Determinantes del acceso al crédito de las PYMES en Colombia", En: Ensayos de economía, vol. 25, núm. 46. [En línea] Disponible en https:// revistas.unal.edu.co/index.php/ede/article/viewFile/53631/53078 recuperado: 22 de enero de 2020

Corabastos (2019a). "Nuestra Historia". Recuperado de https://www.corabastos. com.co/aNuevo/index.php/about-joomla/nuestra-historia

Corabastos (2019b). "App Directorio Comercial", Disponible en https://www. corabastos.com.co/aNuevo/index.php/features/servicios-web/precios-en-linea recuperado: 22 de enero de 2020

Cull, R., Demirgu“ ç?Kunt, A., \& Morduch, J. (2007). “Financial performance and outreach: A global analysis of leading microbanks". En: The Economic Journal, vol. 
117, núm. 517, [En línea] Disponible en https://doi.org/10.1111/j.14680297.2007.02017.x recuperado: 22 de enero de 2020

Escobar, J. D. U. (2013). “El sistema financiero colombiano: estructura y evolución reciente". En: Revista del Banco de la República, vol. 86, núm. 1023. [En línea] Disponible en https:/ / publicaciones.banrepcultural.org/index.php/banrep/ article/view/8421/ 8820 recuperado: 22 de enero de 2020

Espinel, J. A. S., \& Giraldo, L. A. C. (2009). “Evolución del sistema financiero colombiano durante el período 1980-2007". En: Revista de la Facultad de Ciencias Económicas: Investigación y Reflexión, vol. 17, núm 1. [En línea] Disponible en https:/ / www.redalyc.org/pdf/909/90913041002.pdf recuperado: 22 de enero de 2020

Fernandez, J. (2014). Teoría y práctica de la contabilidad (3a. ed.), Madrid, Ediciones pirámide.

Figueroa, H. R. (2016). “Las barreras para acceder al crédito formal dificultan la subsistencia de los microempresarios", En: Revista Finnova: Investigacion e Innovacion Financiera y Organizacional, vol. 2, núm. 3. [En línea] Disponible en https:// doi.org/10.23850/24629758.565 recuperado: 22 de enero de 2020

García, E. A. H., \& Gómez, A. F. O. (2016). “Mercado de crédito informal en Colombia: una aproximación empírica". En: Ensayos de economía, vol. 26, núm. 49. [En línea] Disponible en https:// doi.org/10.15446/ede.v26n49.63820 recuperado: 22 de enero de 2020

Hernandez, R., Fernandez, C. \& Baptista, M. (2010). Metodología de la investigación, Quinta edición, México DF, McGraw Hill

Holguín, P. J. M. (2018). “Inclusión financiera, pero con negación del crédito. Un paso para el "gota a gota". En: Pluriverso, vol. 9, núm. 51. [En línea] Disponible en https:// publicaciones.unaula.edu.co/index.php/Pluriverso/article/view/462 recuperado: 22 de enero de 2020

Massad, C. (2007). Economía para todos, Santiago de Chile, Editorial e Imprenta Maval LTDA.

Mauricio, S. M. S., \& Sandra, R. V. (2009). “Análisis cualitativo y cuantitativo de la informalidad empresarial en Colombia", En: Revista Desarrollo y sociedad, vol. 63, [En línea] Disponible en https:/ / doi.org/10.13043/dys.63.7 recuperado: 22 de enero de 2020

Mballa, L. V. (2017). "Desarrollo local y microfinanzas como estrategias de atención a las necesidades sociales: un acercamiento teórico conceptual”, En: Revista mexicana de ciencias políticas y sociales, vol. 62, núm. 229, México, UNAM, eneroabril. 
Melgarejo, Z., Vera Colina, M., \& Mora Riapira, E. (2013). “Competitividad de la Mipyme y desarrollo regional: estudio del caso colombiano". En: Tendencias, vol. 14, núm. 2. [En línea] Disponible en http://revistas.udenar.edu.co/index.php/rtend/ article/view/1646 recuperado: 22 de enero de 2020

Montoya, C. C. (2001). “El racionamiento de crédito a las microempresas en Colombia. Un estudio de los tipos de racionamiento". En: Coyuntura económica: Investigación económica y social, vol. 41, núm. 1. [En línea] Disponible en https:// www.repository.fedesarrollo.org.co/bitstream/handle/11445/288/Co_Eco_ Sem1_2011_Montoya.pdf?sequence=2\&isAllowed=y recuperado: 22 de enero de 2020

Pesce, G., Esandi, J., Briozzo, A. \& Vigier, H. (2015). “Decisiones de financiamiento en pymes: particularidades derivadas del entrelazamiento empresa-propietario". En: REGE Revista de Gestão, vol. 22, núm. 3. [En línea] Disponible en https:/ / doi.org/ 10.5700/rege567 recuperado: 22 de enero de 2020

Pinto Jiménez, J. de J. (2007). “Sistemas de gestión de competencias basado en capacidades y recursos y su relación con el sistema SECI de gestión del conocimiento realizadas por las pequeñas empresas del Urola Medio (España)". En: Estudios Gerenciales, vol. 23, núm. 105, [En línea] Disponible en https:/ / doi.org/10.1016/ S0123-5923(07)70023-2 recuperado: 22 de enero de 2020

Ramírez, J. P. (2009). Banca y contabilidad, Madrid, Marcial Pons.

Ramírez-Urquid y, M., Mungaray, A., \& Guzmán Gastelum, N. Z. (2009). Restricciones de liquidez en microempresas y la importancia del financiamiento informal en Baja California. En: Región y sociedad, 21(44), 71-90.

Rosende R, F. (1986). “Institucionalidad Financiera y Estabilidad Económica”, En: Cuadernos de Economía, núm. 68, Chile, Instituto de Economía, Pontificia Universidad Católica de Chile, abril.

Superintendencia financiera de Colombia (2019). Conformación del sistema financiero. Disponible en: https://www.superfinanciera.gov.co/jsp/Publicaciones/publicaciones/loadContenidoPublicacion/id/11268/dPrint/1/c/00 recuperado: 22 de enero de 2020

Valencia, G. A. D. (2010). “Las imperfecciones del mercado de créditos, la restricción crediticia y los créditos alternativos". En: Revista CIFE: Lecturas de Economía Social, vol.12, núm. 17, [En línea] Disponible en https:/ / doi.org/10.15332/s22484914.2010.0017.05 recuperado: 22 de enero de 2020 